PROCEEDINGS OF THE

AMERICAN MATHEMATICAL SOCIETY

Volume 126, Number 1, January 1998, Pages 97-103

S 0002-9939(98)04430-X

\title{
EXTENDING THE FORMULA TO CALCULATE THE SPECTRAL RADIUS OF AN OPERATOR
}

\author{
FERNANDO GARIBAY BONALES AND RIGOBERTO VERA MENDOZA
}

(Communicated by Dale Alspach)

\begin{abstract}
In a Banach space, Gelfand's formula is used to find the spectral radius of a continuous linear operator. In this paper, we show another way to find the spectral radius of a bounded linear operator in a complete topological linear space. We also show that Gelfand's formula holds in a more general setting if we generalize the definition of the norm for a bounded linear operator.
\end{abstract}

\section{INTRODUCTION AND BASIC DEFINITIONS}

In all that follows $E$ stands for a linear vector space over the field $\mathbf{C}$ of complex numbers. $E[t]$ will denote a complete locally convex topological vector space, with a Hausdorff topology $t$, and $T: E \rightarrow E$ will be a linear map. Finally, $\vartheta(t)$ will be the filter of all balanced, convex and closed $t$-neighborhoods of zero (in $E$ ).

Definition 1. The linear operator $T: E[t] \rightarrow E[t]$ is said to be a bounded operator, if there is a neighborhood $U \in \vartheta(t)$ such that $T(U)$ is a bounded set.

If in the definition above $T(U)$ is a relatively compact set, then $T$ is said to be a compact operator. Any compact operator is a bounded operator, and any bounded operator is continuous (with the t-topology) (see [5]).

We recall that, given any topological linear space $E[\omega]$ and $S: E[\omega] \rightarrow E[\omega]$ a linear operator, the resolvent of $S$ is the set

$$
\begin{aligned}
\rho_{\omega}(S)=\{\xi \in \mathbf{C} \mid \xi I-S: E[\omega] \rightarrow & E[\omega] \\
& \text { is bijective and has a continuous inverse }\} .
\end{aligned}
$$

The spectrum of $S$ is defined by $\sigma_{\omega}(S)=\mathbf{C} \backslash \rho_{\omega}(S)$ (the set-theoretic complement in $\mathbf{C}$ of the resolvent set), and the spectral radius by

$$
s r_{\omega}(S)=\sup \left\{|\lambda| \mid \lambda \in \sigma_{\omega}(S)\right\} .
$$

Definition 2. A net $\left\{x_{\alpha}\right\}_{J} \subset E$ is said to be $t$-ultimately bounded $(t$-ub) if, given any $V \in \vartheta(t)$, there is a positive real number $r$ and an index $\alpha_{0} \in J$, both depending on $V$, such that $x_{\alpha} \in r V \forall \alpha \geq \alpha_{0}$.

Received by the editors February 27, 1996.

1991 Mathematics Subject Classification. Primary 47A10; Secondary 46 A03.

Key words and phrases. Spectral radius, bounded linear operator, locally convex topological space, $t$-ultimately bounded net.

Research supported by the Coordinación de Investigación Científica de la UMSNH.

(C)1998 American Mathematical Society 
Let us denote by $\Gamma$ the set of all $t$-ub nets in $E$.

Remark 1. Any bounded, convergent or Cauchy net is a $t$-ub net. For more details about $t$-ub nets we refer the reader to [1].

Definition 3. Let $\xi \in \mathbf{C}$. We will say that $\frac{1}{\xi^{n}} T^{n} \stackrel{\Gamma_{t}}{\longrightarrow} 0\left(T^{n}=T \circ T \circ \ldots \circ T, n\right.$ times) if, given both $V \in \vartheta(t)$ and $\left\{x_{\alpha}\right\}_{J} \in \stackrel{\xi^{n}}{\Gamma}$, there exist $\alpha_{0} \in J$ and $n_{0} \in \mathbf{N}$ such that $\frac{1}{\xi^{n}} T^{n}\left(x_{\alpha}\right) \in V \forall \alpha \geq \alpha_{0}$ and $\forall n \geq n_{0}$.

Definition 4. $\gamma_{t}(T)=\inf \left\{|\xi| \mid \frac{1}{\xi^{n}} T^{n} \stackrel{\Gamma_{t}}{\longrightarrow} 0\right\}$.

Remark 2. It is shown by Vera [4] that for a bounded operator T, we have:

(i) $\gamma_{t}(T)<\infty$, and for any $\xi \in \mathbf{C}$ such that $\gamma_{t}(T)<|\xi|, \quad \frac{1}{\xi^{n}} T^{n} \stackrel{\Gamma_{t}}{\longrightarrow} 0$.

(ii) $\operatorname{sr}_{t}(T) \leq \gamma_{t}(T)$, where $\operatorname{sr}_{t}(\mathrm{~T})$ is the spectral radius of $T$.

(iii) When $E[t]$ is a Banach space, $\gamma_{t}(T)=r_{t}(T)$.

In [2] it was proved, based in the above result, that $\gamma_{t}(T)=s r_{t}(T)$ when $T$ is a compact operator. In this paper we extend that result to any bounded operator.

\section{MAIN RESULTS}

From now on let $T: E[t] \rightarrow E[t]$ be a bounded operator and let $U \in \vartheta(t)$ be such that $T(U)$ is a bounded set.

Let $P_{U}$ be the functional of Minkowski (see [3]) generated by $U$, which is a seminorm on $E$. Let $E\left[P_{U}\right]$ denote the vector space $E$ with the topology given by the seminorm $P_{U}$.

Remark 3. The topology on $E$ given by the seminorm $P_{U}$ is coarser than the topology $t\left(P_{U} \leq t\right)$.

Proposition 1. $T: E\left[P_{U}\right] \rightarrow E\left[P_{U}\right]$ is a bounded operator (hence a continuous one).

Proof. Since $T(U)$ is a bounded set and $P_{U} \leq t, T(U)$ is also a $P_{U}$-bounded set in $E\left[P_{U}\right]$.

Definition 5. $\gamma_{P_{U}}(T)=\inf \left\{|\xi| \mid \frac{1}{\xi^{n}} T^{n} \stackrel{\Gamma_{P_{U}}}{\longrightarrow} 0\right\}$.

Here $\Gamma_{P_{U}}$ convergence means that, given any net $\left\{x_{\alpha}\right\}_{J} \subset E$ such that for all $\alpha, P_{U}\left(x_{\alpha}\right) \leq r$ for some positive real number $r$ ( $P_{U}$-bounded net), then $P_{U}\left(\frac{1}{\xi^{n}} T^{n} x_{\alpha}\right) \rightarrow 0$ as a net in $\mathbf{R}$ whose index set is $\mathbf{N} \times \mathbf{J}$.

Proposition 2. $\gamma_{P_{U}}(T)=\gamma_{t}(T)$.

Proof. Let $\xi \in \mathbf{C}$ be such that $\gamma_{P_{U}}(T)<|\xi|$, and let $V \in \vartheta(t)$ and $\left\{x_{\alpha}\right\}_{J} \in \Gamma$ be given. Since $\frac{1}{\xi} T(U)$ is a bounded set, there is a positive real number $r_{1}$ such that $\frac{1}{r_{1} \xi} T(U) \subset V$. In [1] is shown that $\left\{x_{\alpha}\right\}_{J} \in \Gamma \Rightarrow\left\{r_{1} x_{\alpha}\right\}_{J} \in \Gamma$. This implies that there exist both $\alpha_{0} \in J$ and $r_{2}>0$ such that $r_{1} x_{\alpha} \in r_{2} U \forall \alpha \geq \alpha_{0}$, i.e., $P_{U}\left(r_{1} x_{\alpha}\right) \leq r_{2}$, that is, the net $\left\{x_{\alpha}\right\}_{\alpha \geq \alpha_{0}}$ is a $P_{U}$-bounded net; therefore, $\exists \alpha_{1} \in J\left(\alpha_{1} \geq \alpha_{0}\right)$ and $n_{1} \in \mathbf{N}$ such that $P_{U}\left(\frac{1}{\xi^{n}} T^{n}\left(x_{\alpha}\right)\right)<1 \forall \alpha \geq \alpha_{1}, n \geq$ $n_{1}$, that is, $\frac{1}{\xi^{n}} T^{n}\left(x_{\alpha}\right) \in U$ for those indices. Hence

$$
\frac{1}{\xi^{n+1}} T^{n+1} x_{\alpha}=\frac{1}{r_{1} \xi} T\left(\frac{1}{\xi^{n}} T^{n} r_{1} x_{\alpha}\right) \in \frac{1}{r_{1} \xi} T(U) \subset V \quad \forall \alpha \geq \alpha_{1}, n \geq n_{1},
$$


that is, $\frac{1}{\xi^{n}} T^{n} \stackrel{\Gamma_{t}}{\longrightarrow} 0$, and therefore, $\gamma_{t}(T) \leq|\xi|$. This implies that $\gamma_{P_{U}}(T) \leq$ $\gamma_{t}(T)$.

On the other hand, let $\gamma_{t}(T)<|\xi|$ and $\left\{x_{\alpha}\right\}_{J}$, a $P_{U}$-bounded net; that is, $x_{\alpha} \in r U$ for all $\alpha$ and some $r>0$. Then $\left\{\frac{1}{\xi} T x_{\alpha}\right\}_{J} \subset \frac{r}{\xi} T(U)$, where $\frac{r}{\xi} T(U)$ is a $t$-bounded set; therefore, $\left\{\frac{1}{\xi} T x_{\alpha}\right\}_{J} \in \Gamma$. Since $\frac{1}{\xi^{n}} T^{n} \stackrel{\Gamma_{t}}{\longrightarrow} 0$, given $\epsilon>0, \exists \alpha_{0} \in J$ and $n_{0} \in \mathbf{N}$ such that $\frac{1}{\xi^{n+1}} T^{n+1} x_{\alpha}=\frac{1}{\xi^{n}} T^{n}\left(\frac{1}{\xi} T x_{\alpha}\right) \in \epsilon U$ $\forall \alpha \geq \alpha_{0}, n \geq n_{0}$; that is, $P_{U}\left(\frac{1}{\xi^{n+1}} T^{n+1} x_{\alpha}\right) \leq \epsilon$ for those indices. This says that $\frac{1}{\xi^{n}} T^{n} x_{\alpha}$ is $P_{U}$-convergent to 0 ; therefore, $\gamma_{P_{U}}(T) \leq|\xi|$. This implies that $\gamma_{t}(T) \leq \gamma_{P_{U}}(T)$.

\section{Definition 6.}

$$
\begin{gathered}
L(E)=\{S: E[t] \rightarrow E[t] \mid S \text { is a linear and continuous operator }\}, \\
L_{U}(E)=\{S \in L(E) \mid S(U) \text { is a bounded set }\},
\end{gathered}
$$

$L_{U}(E)$ is a vector subspace of the complex vector space $L(E)$.

Remark 4. For the bounded operator $T$ that we have been working on we have $T, T^{n}, \lambda T, \lambda T^{n} \in L_{U}(E)$ for all $n \in \mathbf{N}$ and all $\lambda \in \mathbf{C}$.

Moreover, for any $S \in L(E), S \circ T, T \circ S \in L_{U}(E)$.

Definition 7. For any operator $S \in L_{U}(E)$, we define, taking into account that $S(U)$ is a bounded set, the following real number:

$$
\|S\|_{U}=\sup \left\{P_{U}(S x) \mid x \in U\right\} .
$$

It easy to check that $\left\|S^{n}\right\|_{U} \leq\|S\|_{U}^{n} \forall S \in L_{U}(E)$ and $\forall n \in \mathbf{N}$.

Theorem 1. If $S_{n} \stackrel{\Gamma_{t}}{\longrightarrow} S$ in $L(E)$, then $\left\|S_{n} \circ T-S \circ T\right\|_{U} \rightarrow 0$.

Proof. Let us prove it by way of contradiction.

Let $\epsilon>0$ be such that there exist natural numbers $n_{1}<n_{2}<n_{3}<\ldots$ such that $\epsilon<\left\|S_{n_{k}} \circ T-S \circ T\right\|_{U}$; hence, for each of those $n_{k}$ there is $x_{n_{k}} \in U$ such that $\left.P_{U}\left[\left(S_{n_{k}} \circ T-S \circ T\right) x_{n_{k}}\right)\right]>\epsilon$. Since $\left\{T x_{n_{k}}\right\} \subset T(U)$, it is a bounded sequence; hence, for $V=\epsilon U \in \vartheta(t)$ there is an index $m_{0} \in \mathbf{N}$ such that $\left(S_{n}-S\right)\left(T x_{n_{k}}\right) \in$ $V$ for all $n, n_{k} \geq m_{0}$; this implies that $\left.P_{U}\left[\left(S_{n_{k}} \circ T-S \circ T\right) x_{n_{k}}\right)\right] \leq \epsilon$, which yields a contradiction.

Proposition 3. $\rho_{t}(T) \subset \rho_{P_{U}}(T)$.

Proof. Let us suppose first that $\gamma_{t}(T)<1$. Let $\xi \in \rho_{t}(T)$ be such that $|\xi|>$ $\gamma_{t}(T)$. Then $S=\sum_{k=0}^{\infty} \frac{1}{\xi^{k+1}} T^{k}$ is a continuous operator and $S=(\xi I-T)^{-1}$. Set $S_{n}=\sum_{k=0}^{n} \frac{1}{\xi^{k+1}} T^{k}$. Then $S_{n} \stackrel{\Gamma_{t}}{\longrightarrow} S$, and from Theorem 1 it follows that $\left\|S_{n} \circ \frac{1}{\xi} T-S \circ \frac{1}{\xi} T\right\|_{U} \rightarrow 0$. On the other hand, $S_{n} \circ \frac{1}{\xi} T=S_{n+1}-\frac{1}{\xi} I$ and $S \circ$ $\frac{1}{\xi} T=S-\frac{1}{\xi} I$; hence $\left\|S_{n+1}-S\right\|_{U} \rightarrow 0$. Thereby, given $\left\{x_{m}\right\}_{\mathbf{N}} \subset E$ such that $P_{U}\left(x_{m}\right) \rightarrow 0$, then $P_{U}\left(S x_{m}\right) \leq P_{U}\left[\left(S-S_{n}\right) x_{m}\right]+P_{U}\left(S_{n} x_{m}\right) \rightarrow 0$. This proves that $S: E\left[P_{U}\right] \rightarrow E\left[P_{U}\right]$ is a continuous operator; hence $\xi \in \rho_{P_{U}}(T)$.

Now let $\xi \in \rho_{t}(T)$ be such that $|\xi| \leq \gamma_{t}(T)$. Then $\left|\frac{1}{\xi}\right|>1>\gamma_{t}(T)$, which means that $\frac{1}{\xi} I-T: E\left[P_{U}\right] \rightarrow E\left[P_{U}\right]$ is a continuous operator. Since $\xi I-T=$ 
$\left(\xi-\frac{1}{\xi}\right) I-\left(T-\frac{1}{\xi} I\right)$, we have that

$$
(\xi I-T)^{-1}=\left(\xi-\frac{1}{\xi}\right)^{-1} I \circ\left[\left(T-\frac{1}{\xi} I\right)^{-1}-\left(\frac{1}{\xi}\right)^{-1} I\right] \circ\left(T-\frac{1}{\xi} I\right)^{-1} ;
$$

since the right hand side is the composition of three continuous operators from $E\left[P_{U}\right]$ to $E\left[P_{U}\right]$ we have that $\xi \in \rho_{P_{U}}(T)$.

Finally, let $T$ be such that $\gamma_{t}(T)<r<\infty$. Then $T_{1}=\frac{1}{r} T \in L_{U}(E)$ is such that $\gamma_{t}\left(T_{1}\right)<1$. Hence $\frac{1}{r} \rho_{t}(T)=\rho_{t}\left(T_{1}\right) \subset \rho_{P_{U}}\left(T_{1}\right)=\frac{1}{r} \rho_{P_{U}}(T)$, and therefore $\rho_{t}(T) \subset \rho_{P_{U}}(T)$.

Definition 8. $N=\left\{x \in E \mid P_{U}(x)=0\right\}$.

Remark 5. Since $\left\{x \in E \mid P_{U}(x) \leq 1\right\} \subset U, N \subset U$.

Theorem 2. $N$ is a closed linear subspace of $E$, and $T(x)=0$ for all $x \in N$.

Proof. The first claim follows from the fact that

$$
P_{U}(\xi x+y) \leq|\xi| P_{U}(x)+P_{U}(y) .
$$

For the second claim let's take $x \in N$; then $m x \in N$ for $m=1,2, \ldots$. Let $V$ be any balanced, convex and closed $t$-neighborhood of 0 . Since $\{m T(x)\}_{m=1,2,3, \ldots} \subset$ $T(N) \subset T(U)$ and the latter set is bounded, there exists $r \in \mathbf{R}^{+}$such that $\{m T(x)\} \subset r V \Rightarrow T(x) \in \frac{r}{m} V \subset V$ when $m>r$. Since $V$ was an arbitrary neighborhood of zero and $E[t]$ is Hausdorff, then $T(x)=0$.

Definition 9. Let $E / N$ be the quotient linear space and let $\hat{P}_{U}$ be the norm on it defined by $\hat{P}_{U}(x+N)=P_{U}(x)$ (see [3]).

Remark 6. $(E / N)\left[\hat{P}_{U}\right]$ will denote the vector space $E / N$ with the topology given by the norm $\hat{P}_{U}$.

Definition 10. Let $\hat{T}:(E / N) \rightarrow(E / N)$ be defined by $\hat{T}(x+N)=T(x)+N$.

Remark 7. It is easy to show that $\hat{T}$ is a well defined linear map.

Proposition 4. $\hat{T}:(E / N)\left[\hat{P}_{U}\right] \rightarrow(E / N)\left[\hat{P}_{U}\right]$ is a linear and bounded operator (hence $\hat{T}$ is continuous).

Proof. $U / N$ is the unit ball in $(E / N)\left[\hat{P}_{U}\right]$ and $\hat{T}(U / N)=(T(U)+N) / N$. The latter set is $\hat{P}_{U}$-bounded because the canonical projection $E\left[P_{U}\right] \rightarrow(E / N)\left[\hat{P}_{U}\right]$ is a continuous map.

Remark 8. Since $(E / N)\left[\hat{P}_{U}\right]$ is a norm space we can define, as usual, the norm of $\hat{T}$, and this will be denoted by $\|\hat{T}\|_{\hat{P}_{U}}$.

Proposition 5. $\gamma_{\hat{P}_{U}}(\hat{T})=\gamma_{P_{U}}(T)$.

Proof. Set $|\xi|>\gamma_{P_{U}}(T)$. Let $\left\{x_{\alpha}+N\right\}_{J}$ be a $\hat{P}_{U}$-bounded net in $E / N$; then $\left\{x_{\alpha}\right\}_{J}$ is a $P_{U}$-bounded net in $E$; hence, given $\epsilon>0$, there are indices $\alpha_{0} \in J$ and $n_{0} \in \mathbf{N}$ such that $\frac{1}{\xi^{n}} T^{n} x_{\alpha} \in \epsilon U \forall \alpha \geq \alpha_{0}$ and $n \geq n_{0}$. Thus

$$
\frac{1}{\xi^{n}} \hat{T}^{n}\left(x_{\alpha}+N\right)=\frac{1}{\xi^{n}} T^{n} x_{\alpha}+N \in \epsilon(U / N), \quad \alpha \geq \alpha_{0}, n \geq n_{0}
$$

This implies that $\gamma_{\hat{P}_{U}}(T) \leq|\xi|$. Hence $\gamma_{\hat{P}_{U}}(T) \leq \gamma_{P_{U}}(T)$. 
Set $|\xi|>\gamma_{\hat{P}_{U}}(T)$. Let $\left\{x_{\alpha}\right\}_{J}$ be a $P_{U}$-bounded net in $E$. Then $\left\{x_{\alpha}+N\right\}_{J}$ is a $\hat{P}_{U}$-bounded net in $E / N$; hence, given $\epsilon>0$, there are indices $\alpha_{0} \in J$ and $n_{0} \in \mathbf{N}$ such that $\frac{1}{\xi^{n}} \hat{T}^{n}\left(x_{\alpha}+N\right) \in \epsilon(U / N) \forall \alpha \geq \alpha_{0}, n \geq n_{0}$. This implies that for those indices $\frac{1}{\xi^{n}} T^{n} x_{\alpha}=\epsilon u_{\alpha}+z_{\alpha}, u_{\alpha} \in U, z_{\alpha} \in N$; hence $P_{U}\left(\frac{1}{\xi^{n}} T^{n} x_{\alpha}\right) \leq P_{U}\left(\epsilon u_{\alpha}\right)+P_{U}\left(z_{\alpha}\right) \leq \epsilon+0=\epsilon$, and thus $|\xi|>\gamma_{P_{U}}(T)$. This implies that $\gamma_{\hat{P}_{U}}(T) \geq \gamma_{P_{U}}(T)$.

Proposition 6. $\rho_{P_{U}}(T)=\rho_{\hat{P}_{U}}(\hat{T})$.

Proof. $\xi \in \rho_{P_{U}}(T) \Rightarrow \xi I-T: E\left[P_{U}\right] \rightarrow E\left[P_{U}\right]$ is bijective and has a continuous inverse.

Let us show that $A:(E / N)\left[\hat{P}_{U}\right] \rightarrow(E / N)\left[\hat{P}_{U}\right]$ defined by $A(x+N)=$ $(\xi I-T)^{-1}(x)+N$, which is a linear and continuous map, is the inverse function of $\xi \hat{I}-\hat{T}$. For this, $A(\xi \hat{I}-\hat{T})(x+N)=A(\widehat{\xi I-T})(x+N)=A((\xi I-T)(x)+N)=$ $(\xi I-T)^{-1}(\xi I-T)(x)+N=x+N$. In a similar way it can be proved that $(\xi \hat{I}-\hat{T}) \circ A=I$. This implies that $\xi \in \rho_{\hat{P}_{U}}(\hat{T})$.

It is just routine to prove the set contention in the other way around.

Definition 11. $\widetilde{(E / N)}\left[\tilde{P}_{U}\right]$ will denote the completion (as a normed space) of $(E / N)\left[\hat{P}_{U}\right]$, and $\tilde{T}$ will denote the natural extension of $\hat{T}$.

Remark 9. $\widetilde{(E / N)}\left[\tilde{P}_{U}\right]$ is a Banach space. Besides, since $\hat{T}$ is a bounded operator, $\tilde{T}: \widetilde{(E / N)}\left[\tilde{P}_{U}\right] \rightarrow \widetilde{(E / N)}\left[\tilde{P}_{U}\right]$ is a bounded operator (see [3]).

Remark 10. Since $\widetilde{(E / N)}\left[\tilde{P}_{U}\right]$ is a Banach space we can define, as usual, the norm of $\tilde{T}$; this will be denoted by $\|\tilde{T}\|_{\tilde{P}_{U}}$.

Proposition 7. $\gamma_{\hat{P_{U}}}(\hat{T})=\gamma_{\tilde{P_{U}}}(\tilde{T})$.

Proof. Since $\tilde{T}$ is an extension of $\hat{T}$, the proof follows immediately from the definitions of $\gamma_{\hat{P_{U}}}(\hat{T})$ and $\gamma_{\tilde{P_{U}}}(\tilde{T})$.

Proposition 8. $\rho_{\hat{P_{U}}}(\hat{T})=\rho_{\tilde{P_{U}}}(\tilde{T})$.

Proof. If $\xi \in \rho(\hat{T})$, then $\xi I-\hat{T}:(E / N)\left[\hat{P_{U}}\right] \rightarrow(E / N)\left[\hat{P}_{U}\right]$ is bijective and has a continuous inverse, so that both $\xi I-\hat{T}$ and $(\xi I-\hat{T})^{-1}$ have a continuous extension to $\widetilde{(E / N)}$, which are precisely $\xi I-\tilde{T}$ and $(\xi I-\tilde{T})^{-1}$ respectively. This implies that $\xi \in \rho(\tilde{T})$.

On the other hand, if $\xi \in \rho(\tilde{T})$, then $\xi I-\tilde{T}: \widetilde{(E / N)}\left[\tilde{P_{U}}\right] \rightarrow \widetilde{(E / N)}\left[\tilde{P}_{U}\right]$ is bijective and has a continuous inverse; hence the restrictions of those functions to $(E / N)\left[\hat{P_{U}}\right]$ are precisely $\xi I-\hat{T}$ and its inverse function, which are continuous functions for being the restrictions of continuous ones. Then $\xi \in \rho(\hat{T})$.

Theorem 3. $\gamma_{t}(T)=s r_{t}(T)$.

Proof. By Remark 2 (ii) it suffices to show that $s r_{t}(T) \geq \gamma_{t}(T)$. Also, from Remark 2 (iii) we get

$$
s r_{\tilde{P_{U}}}(\tilde{T})=\gamma_{\tilde{P_{U}}}(\tilde{T})
$$


because $\widetilde{(E / N)}\left[\tilde{P_{U}}\right]$ is a Banach space. From Propositions 2, 5 and 7 we obtain

$$
\gamma_{t}(T)=\gamma_{\tilde{P}_{U}}(\tilde{T})
$$

From Propositions 3, 6 and 8 we obtain

$$
\rho_{t}(T) \subset \rho_{\tilde{P_{U}}}(\tilde{T})
$$

this implies that

$$
s r_{\tilde{P}_{U}}(\tilde{T}) \leq s r_{t}(T) .
$$

From (1), (2) and (3) we finally get

$$
\gamma_{t}(T) \leq s r_{t}(T)
$$

\section{A generalization of Gelfand's formula}

In this part we prove that Gelfand's formula (see [3]) applies for a bounded operator defined on a topological vector space. Following the notation from the sections above, we will show that we can use $\|T\|_{U}$ in Gelfand's formula to calculate the spectral radius of $T$.

Proposition 9. For any $T \in L_{U}(E),\|T\|_{U}=\|\hat{T}\|_{\hat{P}_{U}}$.

Proof. Set $r>\|T\|_{U}$. Then $T(U) \subset r U$; hence $P_{U}(T x) \leq r$ for all $x \in U$. This implies that $\|\hat{T}\|_{\hat{P}_{U}} \leq r$, and therefore $\|\hat{T}\|_{\hat{P}_{U}} \leq\|T\|_{U}$.

Set $r<\|T\|_{U}$. Then there exists $x \in U$ such that

$$
r<P_{U}(T x)=\hat{P}_{U}(\hat{T}(x+N)) \leq\|\hat{T}\|_{\hat{P}_{U}} .
$$

This implies that $\|\hat{T}\|_{\hat{P}_{U}}=\|T\|_{U}$.

Corollary 1. $\left.\left\|\left.\tilde{T}\right|_{\tilde{P}_{U}}=\right\| \hat{T}\right|_{\hat{P}_{U}}=\|T\|_{U}$.

Theorem 4. $\operatorname{sr}_{t}(T)=\lim _{n \rightarrow \infty}\left\|T^{n}\right\|_{U}^{\frac{1}{n}}$ for any $T \in L_{U}(E)$.

Proof. We recall first that $T \in L_{U}(E) \Rightarrow T^{n} \in L_{U}(E)$. From (1), (2), and Theorem 3 we obtain

$$
s r_{t}(T)=s r_{\tilde{P}_{U}}(\tilde{T}) .
$$

Because $\widetilde{(E / N)}\left[\tilde{P_{U}}\right]$ is a Banach space, Gelfand's formula holds:

$$
s r_{P_{U}}(\tilde{T})=\lim _{n \rightarrow \infty}\left\|\tilde{T}^{n}\right\|^{\frac{1}{n}} .
$$

Finally, using (4) and (5) and the corollary above, we obtain

$$
s r_{t}(T)=\lim _{n \rightarrow \infty}\left\|T^{n}\right\|_{U}^{\frac{1}{n}} .
$$




\section{REFERENCES}

[1] C. L. DeVito, On Alaoglu's Theorem, Bornological Spaces and the Mackey-Ulam Theorem. Math. Ann. 192, 83-89 (1972). MR 44:2014

[2] F. Garibay and R. Vera,A Formula to Calculate the Spectral Radius of a Compact Linear Operator. Internat J. Math. \& Math. Sci. 20, no. 3 (1997), 585-588.

[3] W. Rudin, Functional Analysis. New York, McGraw-Hill, Inc. 1991. MR 92k:46001

[4] R. Vera, Linear Operators on Locally Convex Topological Vector Spaces. Ph.D. Thesis, Department of Mathematics, University of Arizona, 1994.

[5] K. Yosida, Functional Analysis. Springer Verlag, New York, 1965. MR 31:5054

Escuela de Ciencias Físico-Matemáticas, Universidad Michoacana de San Nicolás de Hidalgo, Edificio B, Planta Baja, Ciudad Universitaria, Morelia, Michoacán, CP 58060 , MÉXICO

E-mail address: fgaribay@zeus.ccu.umich.mx

E-mail address: rvera@zeus.ccu.umich.mx 